\title{
A Noncontact Picolitor Droplet Handling by Photothermal Control of Interfacial Flow
}

\author{
Masakazu Muto, Makoto Yamamoto, and Masahiro MotosuKE ${ }^{\dagger}$ \\ Department of Mechanical Engineering, Tokyo University of Science, 6-3-1 Niijuku, Katsushika, \\ Tokyo 125-8585, Japan
}

\begin{abstract}
We present a noncontact handling of droplets in a microfluidic platform by the Marangoni convection, interfacial tension driven flow, generated by a light-induced local temperature gradient in the surrounding liquid of the droplet. Droplets flowing in a microchannel experience a force due to the interfacial tension gradient when approaching the heated area. This method provides noncontact, selective and flexible manipulation for droplets flowing in microchannel network. In this study, an $\mathrm{O} / \mathrm{W}$ emulsion system with oleic acid for the dispersed phase and a buffer solution for the continuous one was used. Trajectory control and trapping for droplets with $5-65 \mathrm{pL}$ in volume was achieved by patterned laser irradiation. Also, we quantitatively evaluated the driving force exerted on droplets by measuring the fluidic temperature distribution around the droplet. From the balance of the drag force and the photo-induced Marangoni force, the driving force was determined using the measured temperature gradient of the droplet. From the results, the applicability of noncontact droplet manipulation using the photothermal Marangoni effect by continuous-phase heating has been demonstrated.
\end{abstract}

Keywords Microfluidics, droplet, photothermal effect, Marangoni convection, interfacial tension, continuous phase heating, laser-induced fluorescence

(Received October 31, 2015; Accepted November 24, 2015; Published January 10, 2016)

\section{Introduction}

Recently, there has been a growing interest in microfluidic technology owing to its highly diverse applications, from point-of-care diagnostics to microreactors. An approach based on multiphase flow, so-called droplet-based microfluidics, focuses on the use of discrete volumes of fluid involving cells and reagents in the dispersed phase. ${ }^{1,2}$ Droplet-based microfluidics has advantages in the generation of monodisperse droplets, ${ }^{3,4}$ rapid reagent mixing due to interfacial flow, ${ }^{5}$ and single-cell analysis in a confined droplet reactor. ${ }^{6,7}$ Additionally, droplet manipulation techniques have been proposed for advanced droplet-based analysis; dielectrophoresis, ${ }^{8}$ magnetic field, ${ }^{9}$ optical vortex ${ }^{10}$ are used. Among them, an optical method would provide beneficial performance with noncontact and remote handling without contamination and on-chip fabrication.

Since the effects of interfacial phenomena on the fluidic behavior become dominant with decreasing of the length scale, as in microfluidic devices, ${ }^{11}$ the local control of interfacial tension can be effective for the handling of droplets. When the temperature gradient of the surrounding fluid is generated in the vicinity of a droplet, Marangoni convection, an interfacial flow induced by an imbalance of the interfacial tension, occurs, which results in a pressure difference around the droplet. Eventually, the driving force works on the droplet, and moves it toward an area with lower pressure, that is, lower interfacial

$\dagger$ To whom correspondence should be addressed.

E-mail: mot@rs.tus.ac.jp tension. Figure 1 shows a schematic of droplet manipulation by a thermally activated gradient of interfacial tension. When the temperature gradient is induced by a focused laser beam, flexible and selective droplet handling can be performed. ${ }^{12-15}$ This photothermal droplet handling method has following characteristics: (i) remote access to the fluidic system without any further micro/nano-fabrication, (ii) contamination-free and noncontact manipulation, (iii) high selectivity of handling, and (iv) minimum pressure loss due to no obstacles in the channel. Liquid-liquid interfacial tension normally has a negative temperature coefficient resulting in the interfacial flow to the

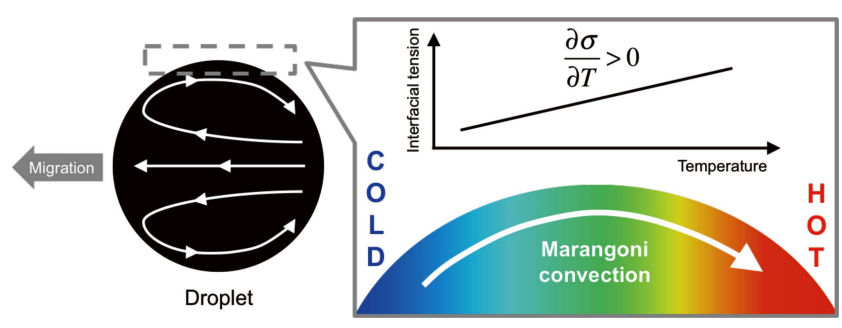

Fig. 1 Schematic of droplet migration by the Marangoni effect under a positive temperature coefficient of the interfacial tension $(\partial \sigma / \partial T>0)$. Under the temperature gradient, the interfacial flow occurs from a low-tension area to a high-tension area. Eventually the droplet moves away from the hot area because of a pressure difference around the droplet due to Marangoni convection. In this study, a local temperature gradient generated by the laser irradiation enabled selective and flexible handling of the droplet. 


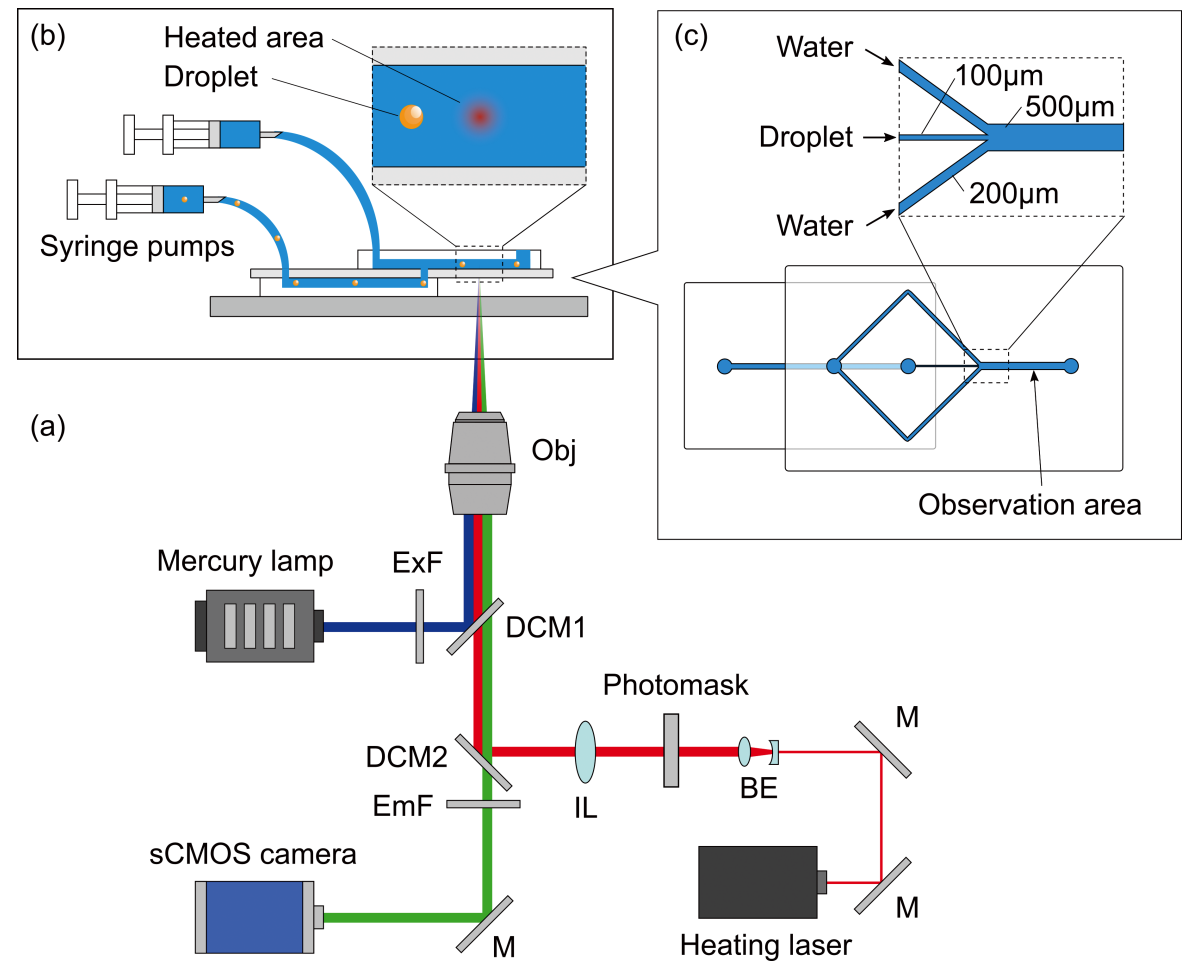

Fig. 2 Schematic diagram of (a) the heating optical setup with a reduced-projection exposure optics and the measurement setup, (b) a sectional view of the microfluidic device, and (c) a top view of the device. The photothermal droplet manipulation and the temperature measurement by LIF are simultaneously possible in this system. The droplet behavior under laser irradiation in the microfluidic chip is observed, and the image taken by a sCMOS camera is converted to the temperature profile using a temperature-sensitive fluorescent dye.

cooler area. Also, it is known that dynamic transport of the surfactant would affect the net tension balance and reverse the direction of interfacial flow. ${ }^{13}$ The interface in the present system has a positive temperature coefficient of the tension with the aid of a surfactant. The interfacial flow to the hotter area makes the droplet migrate away from the heating light position, as in Fig. 1. In our study, the temperature gradient around the droplet is generated by the absorption of light energy irradiated in the surrounding liquid, so that the heating pattern can be easily controlled with a high spatial- and temporal-resolution. The heating of the surrounding fluid as a trigger of droplet migration provides a wider controllable area due to thermal diffusion in the liquid, compared with the case in which the focused laser directly heats each droplet in the chip. ${ }^{12,13}$ Estimating the photothermal manipulation force exerted on each droplet is also of interest. The force was previously evaluated as a trapping force balanced with the flow resistance using a bypassed channel. ${ }^{13}$ However, it would be insufficient in the force estimation without the temperature information in order to design a droplet microfluidic system under photothermal control.

This article provides an overview of our experimental work of droplet handling relying on the photothermal Marangoni convection caused by optical heating of the surrounding liquid of the droplet. A temperature field measurement based on laser-induced fluorescence (LIF) $)^{16,17}$ and theory of force estimation based on the measured temperature distribution around the droplet under light irradiation is also presented. Finally, the dependences of several parameters on the manipulation force for a picoliter droplet and an analysis of the movement in controlled droplets under laser heating are shown.

\section{Experimental}

Reagents and chemicals

In our experiments, an $\mathrm{O} / \mathrm{W}$ emulsion system with oleic acid for the droplet (dispersed phase) and a tetraborate $\mathrm{pH}$ standard buffer solution (205-08775, Wako Pure Chemical Industries) for the surrounding fluid (continuous phase) was employed. Since both liquids do not have a light absorption band in visible wavelengths, a dye substance as the absorption enhancer was added only in the continuous phase. In this study, 4.0, 5.0 and $10.0 \mathrm{mM}$ of Brilliant Blue FCF $\left(\mathrm{C}_{37} \mathrm{H}_{34} \mathrm{~N}_{2} \mathrm{Na}_{2} \mathrm{O}_{9} \mathrm{~S}_{3}\right)$, a well-known food color, was used. Additionally, in order to measure the temperature distribution of the surrounding fluid around the droplets by LIF, $5.0 \mathrm{mM}$ of fluorescein as a temperature-sensitive fluorophore was also added into the continuous phase. The buffer solution has a constant $\mathrm{pH}$ of 9.18 (at $25^{\circ} \mathrm{C}$ ) to prevent the effect of a pH change on the fluorescent emission of fluorophore. Also, a nonionic surfactant of Tween ${ }^{\circledR}$ 20 with a concentration of $10.0 \mathrm{mM}$ was added to stabilize the droplet interface. The droplet was obtained with a stirrer $(1500 \mathrm{rpm}, 20 \mathrm{~s})$. The size of the droplets in this study ranged from 10 to $50 \mu \mathrm{m}$ in diameter, namely 5 to $65 \mathrm{pL}$ in volume.

\section{Apparatus}

Figure 2(a) shows a schematic of the heating optical system used to induce a local interfacial tension gradient around droplets in a microfluidic device using reduced-projection exposure optics. The heating optical system was modified from our previous setup without any projection functionality. ${ }^{18}$ As for 


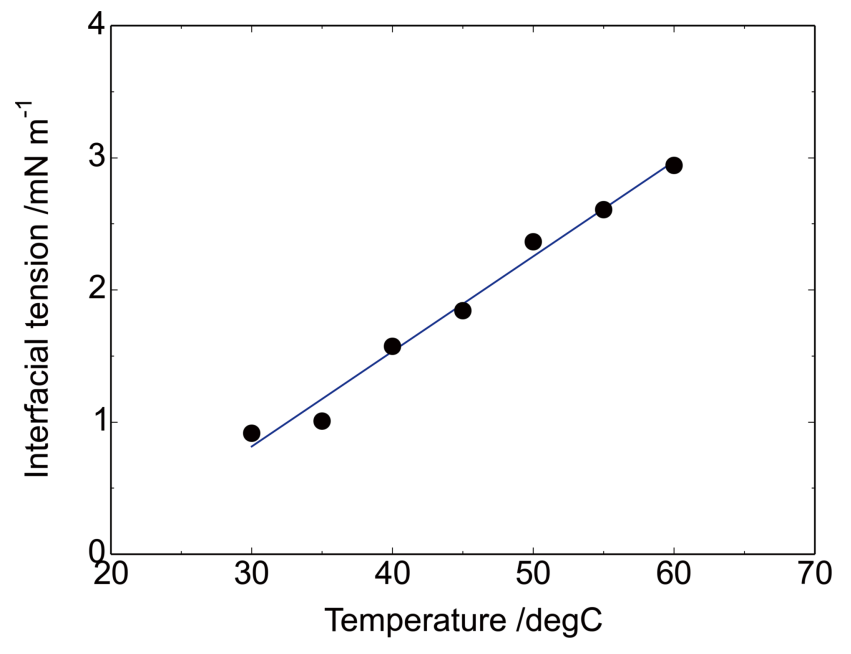

Fig. 3 Interfacial tension as a function of the temperature for water in oleic acid measured by the pendant drop method. The interfacial tension in this system shows a positive temperature coefficient of $7.2 \% / \mathrm{K}$.

the reduced-projection system, laser light is irradiated into liquid in a fluidic channel after forming an irradiation pattern characterized by a photomask. The reduced-projection optical system was built in an inverted microscope so as to achieve a co-axial illumination having the same optical paths in both the illumination and the observation. The heating optical system consists of a diode laser with a wavelength of $635 \mathrm{~nm}$ (Heating laser), a beam expander (BE), the photomask, and an imaging lens (IL). The laser beam was expanded by BE, passed IL, and irradiated into the microchannel. The fluorescent image was recorded by a scientific complementary metal-oxide semiconductor $(\mathrm{sCMOS})$ camera $(1920 \times 1440$ pixels $)$. A dichroic mirror 1 (DCM 1) was designed to pass the heating laser beam and fluorescence to perform the LIF measurement and to reflect the excitation wavelength for the fluorescent dye from a mercury lamp. A dichroic mirror 2 (DCM 2) was designed to pass the fluorescence and to reflect the heating laser beam. An objective lens $(\mathrm{Obj})$ used in the present study had a magnification of 20 and NA of 0.45 .

The interfacial tension at different temperatures was measured by a pendant drop method. ${ }^{19}$ Water with a surfactant was suspended from a fine needle of $22 \mathrm{G}$ in bulk oleic acid. The temperature of the container for the bulk liquid was controlled by a thermostat with a water-circulator. The interfacial tension was determined from the shadow image recorded by a chargecoupled device (CCD) camera in the temperature range from 30 to $60^{\circ} \mathrm{C}$. During the measurement, the temperature of the container for the bulk liquid was kept constant so as to prevent any temperature gradient around the suspended drop.

\section{Fluidic device}

Figure 2(b) depicts the microfluidic device used in the present study. The device consists of two PDMS (polydimethylsiloxane) channels fabricated by softlithograpy ${ }^{20}$ and a glass plate between them. Droplets were seeded from a bottom channel with $200 \mu \mathrm{m}$ in width and $50 \mu \mathrm{m}$ in height to a top channel. This two-layer injection system of droplets was employed to ensure a stable seeding of droplets into the observation channel. ${ }^{18}$ The top fluidic channel employs sheath flow (Fig. 2(c)) to control the position and speed of droplets suitable for the manipulation and measurement. The channel widths of the droplet inlet,

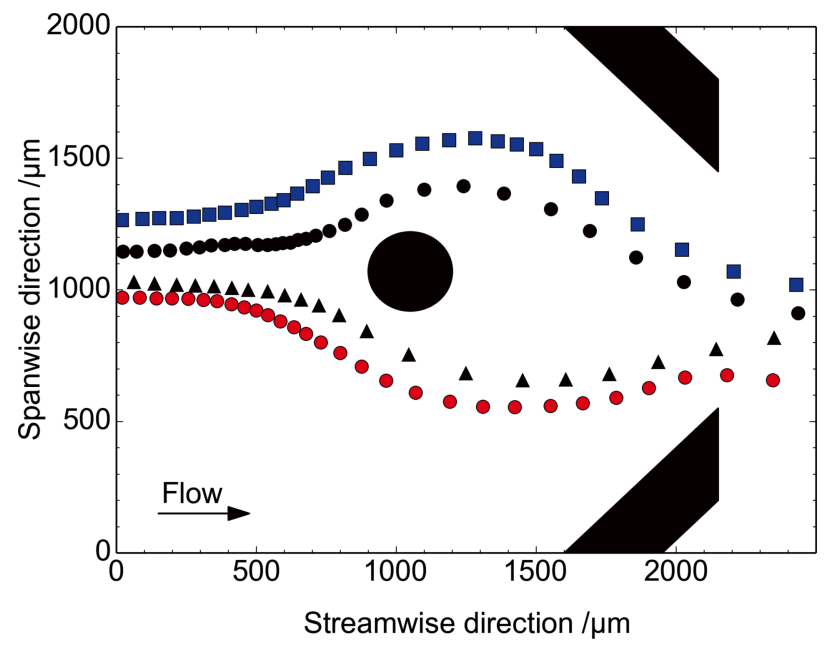

Fig. 4 Trajectories of several droplets under patterned laser irradiation. An oleic acid droplet flows so as to avoid the irradiated light pattern (hot area) due to photothermally activated Marangoni flow. The black pattern is the light pattern irradiated.

water sheath flow and observation were 100, 200 and $500 \mu \mathrm{m}$, respectively. The height of the top channel was the same as the bottom one. Irradiation of the heating light was carried out $1000 \mu \mathrm{m}$ downstream of the junction. All of the inlets were connected to separate syringe pumps. The device was placed on two Peltier elements connected to a water-circulated heat sink controlled by a thermostat. An adhesive thermal-conductive sheet was used to reduce the thermal resistance between the device and the Peltier elements. A K-type thermocouple with a thickness of $40 \mu \mathrm{m}$ was attached to the microchannel so as to obtain the reference temperature of the device used in a calibration procedure of LIF.

\section{Results and Discussion}

Interfacial tension with a temperature dependence

Interfacial tension measurements show the results in Fig. 3. The tension indicates a positive temperature coefficient of $7.2 \% / \mathrm{K}$ in the temperature range from 30 to $60^{\circ} \mathrm{C}$. This positive temperature dependence has been seen in some liquid alloy ${ }^{21}$ or alcohol aqueous solution ${ }^{22}$ in a certain temperature range. Also, there is a possibility that a slight temperature gradient in the container for the bulk liquid due to heat conduction via the needle for the drop would induce weak transport of the surfactant. In either case, the droplet under light irradiation existed with the temperature gradient during handling in the present study. Therefore, we utilized the temperature coefficient of the interfacial tension measured in this experiment for a following calculation of the driving force.

\section{Experimental validation of photothermal droplet handling}

The potential of the photothermal Marangoni effect for parallel and multiple sorting of droplets was investigated using patterned light irradiation. The photomask characterizing the heating domain has an aperture with a circle and two inclined bars. The flow velocity upstream of the heating area was $55 \mu \mathrm{m} / \mathrm{s}$ and the laser power measured close to the microfluidic device was around $100 \mathrm{~mW}$. As shown in Fig. 4, droplets flow while changing their trajectories as if they avoid the irradiated light pattern. This movement can be induced due to the 
interfacial flow under a positive temperature coefficient in the interfacial tension. Because the continuous phase was heated, a wide controllable area was obtained compared with the dispersed-phase heating. ${ }^{12,13,15}$ In this system, an oleic acid droplet less than $10 \mu \mathrm{m}$ in diameter could not be manipulated with sufficiently high controllability. In order to determine the manipulation force in the platform, temperature measurements in the surrounding liquid under light irradiation were performed.

Establishment of the temperature-measurement system with laser-induced fluorescence

When measuring the temperature distribution of liquid around the droplets with laser heating by LIF, the calibration curve between the liquid temperature and the fluorescent intensity is needed. Since the accuracy of the temperature measurement by LIF is determined by the calibration, it is essential to secure a precise calibration process. In the calibration process, the fluorescent intensity was obtained in a temperature range from 20 to $60^{\circ} \mathrm{C}$ with repetition in both the heating and cooling processes to confirm no hysteresis. The fluorescent intensity was determined by the mean value on the central $200 \times 200$ pixels over 20 images successively recorded at $1 \mathrm{~ms}$ intervals. To prevent quenching of the fluorophore during the calibration process, the fluid kept being seeded with a syringe pump. We also recorded 20 images for a background image without any

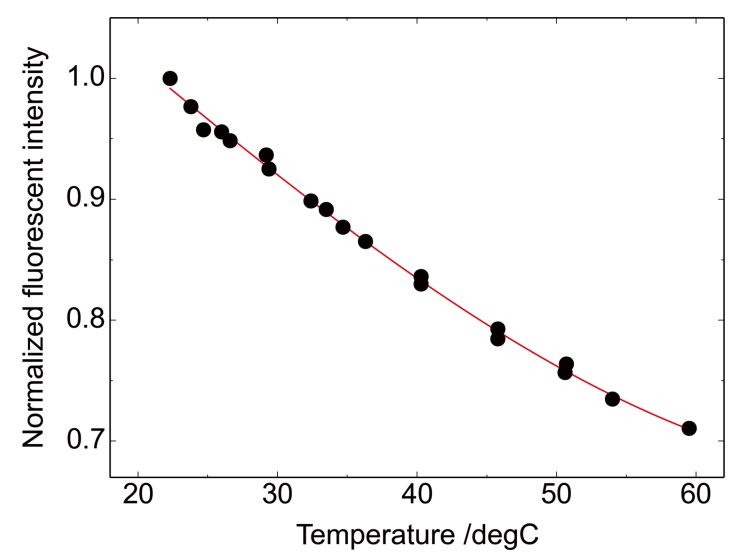

Fig. 5 Calibration curve between the temperature and the fluorescent intensity of the working liquid. The intensity is normalized by that at room temperature. The fluorescent intensities show no hysteresis sliding up and down the temperature controlled by the Peltier modules. laser heating so as to remove inherent noise due to the dark-current noise or stray light. Also, the intensity after subtracting the background noise was normalized by that at room temperature $\left(22.5^{\circ} \mathrm{C}\right)$.

From the above, we obtained the optimized calibration curve of the working fluid with a surfactant, absorbing dye in the buffer solution with the fluorescence dye, as shown in Fig. 5. The fluorescent intensity showed a negative temperature dependence, approximately -0.8 to $-1 \% / \mathrm{K}$ in this range. The normalized fluorescent intensity as cubic polynomials of the temperature (unit in $\mathrm{K}$ ) can be expressed as follows:

$$
\begin{aligned}
I(T)=8.417 \times 10^{-7} T^{3}-3.662 \times 10^{-5} T^{2}- \\
9.098 \times 10^{-3} T+1.203 .
\end{aligned}
$$

The liquid temperature can be obtained using the inversed function of Eq. (1).

The surfactant concentration indicated no influence on the fluorescent intensity. Figure 6 indicates the temperature fields with different concentrations of absorbing dye under the same light-irradiation condition; $5.0 \mathrm{mM}$ was employed in subsequent experiments.

\section{Size dependent droplet handling}

An experiment was performed using a circular irradiation pattern with a $200-\mu \mathrm{m}$ diameter. The laser power was around $200 \mathrm{~mW}$. The trajectories of droplets with different sizes influenced by the photothermal Marangoni effect are shown in Fig. 7. The time intervals in each plot were $50 \mathrm{~ms}$, and the flow rates of the continuous phase and dispersed phase were 100 and $30 \mu \mathrm{L} / \mathrm{h}$, respectively. The temperature distribution during laser heating measured by LIF overlaps in the same figure. Note that there was no significant distortion in the temperature profile when the droplet passed close to the heated area. This means that the influence of the Marangoni convection generated at the liquid-liquid interface of the droplet is negligibly small due to the low Peclet number, ratio of convectional heat transfer and thermal diffusion, in the present condition.

Figure 8 illustrates the size-dependent migration displacements in the spanwise direction. This result clearly shows that a larger droplet has an increased displacement from the heated area. In the same temperature gradient, a large temperature difference is generated at both ends of a large droplet, resulting in a stronger driving force by the Marangoni convection exerted on the droplet. We qualitatively predict that the driving force acting on the droplet, $F_{\mathrm{M}}$, depends on the temperature dependence of the interfacial tension, the temperature gradient, and the surface (a)

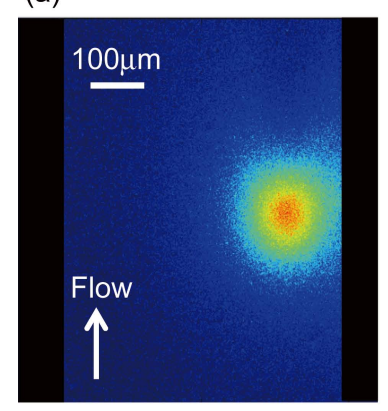

(b)

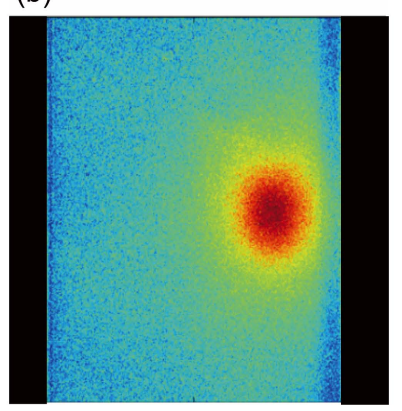

(c)

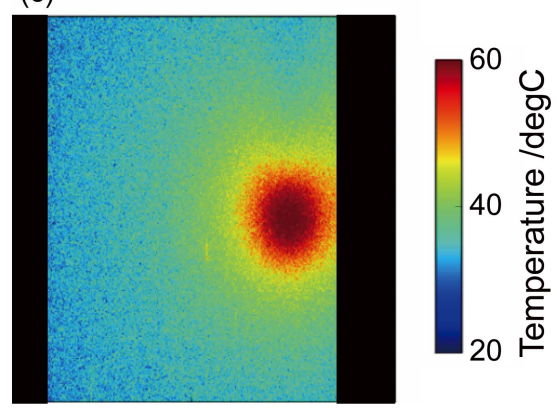

Fig. 6 Temperature distribution under the same light irradiation condition with the absorbing dye concentration of (a) 4.0, (b) 5.0, and (c) $10.0 \mathrm{mM}$. The difference of the absorbing dye concentration results in the difference of the temperature fields. 

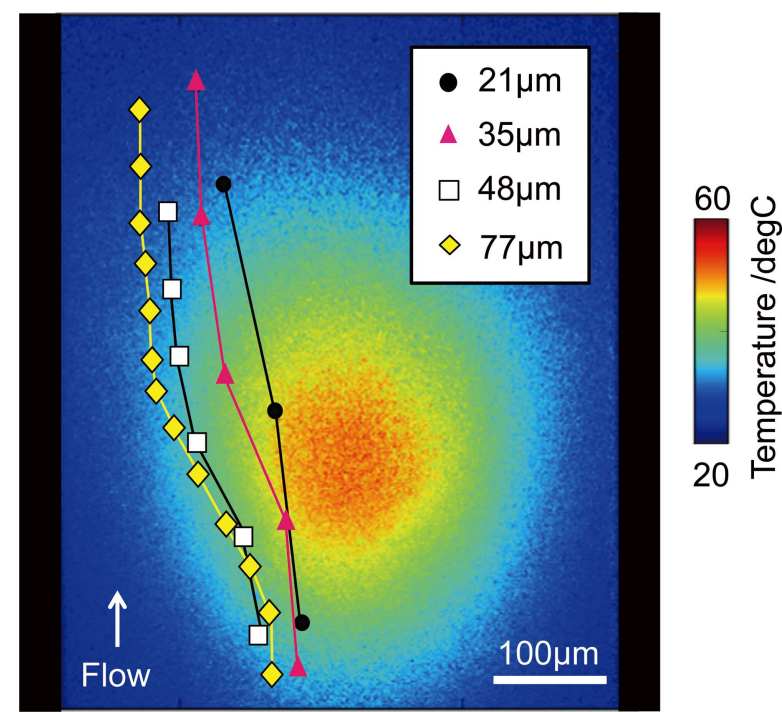

Fig. 7 Trajectory of droplets with different sizes under laser heating with a $200-\mu \mathrm{m}$ circular pattern. The temperature distribution induced by the laser heating measured by LIF is also in the same figure. Droplet flows as avoiding the heated area due to photothermal Marangoni convection.

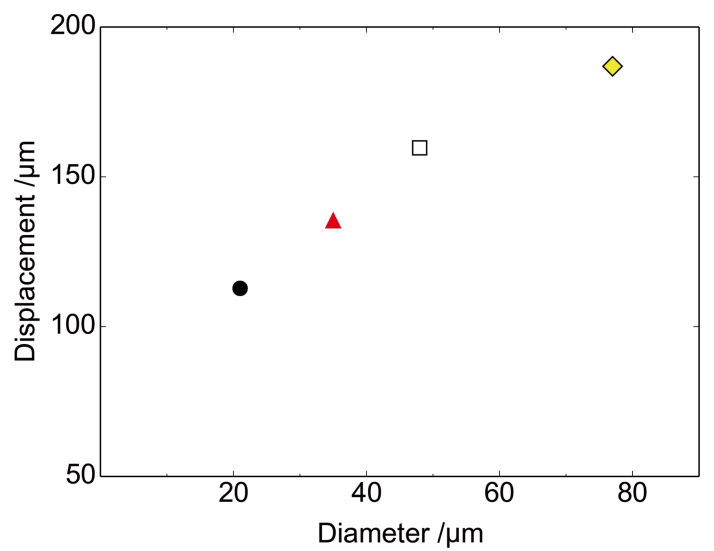

Fig. 8 Size dependence of the spanwise displacement in Fig. 7. The larger droplet shows a greater displacement because of the larger tension difference induced by laser heating.

area of droplet, as presented in the following equation:

$$
F_{\mathrm{M}} \propto \frac{\partial \sigma}{\partial T} \cdot \frac{\partial T}{\partial x} \cdot d^{2}=\frac{\partial \sigma}{\partial T} \cdot \Delta T \cdot d,
$$

where $\partial \sigma / \partial T$ is the temperature coefficient of the interfacial tension, $\partial T / \partial x$ is the temperature gradient around the droplet, and $d$ is the droplet diameter. Here, $\partial T / \partial x$ is converted to $\Delta T / d$, the temperature difference between both sides of the droplet along the temperature gradient, which would be an easily measurable value by LIF. A detailed description of the drivingforce estimation is presented in the next section.

\section{Quantification of the manipulation force for a droplet}

A quantitative estimation of the driving force acting on a droplet by the photothermal Marangoni convection is presented in this section. Here, we assume that the fluidic motion is in the

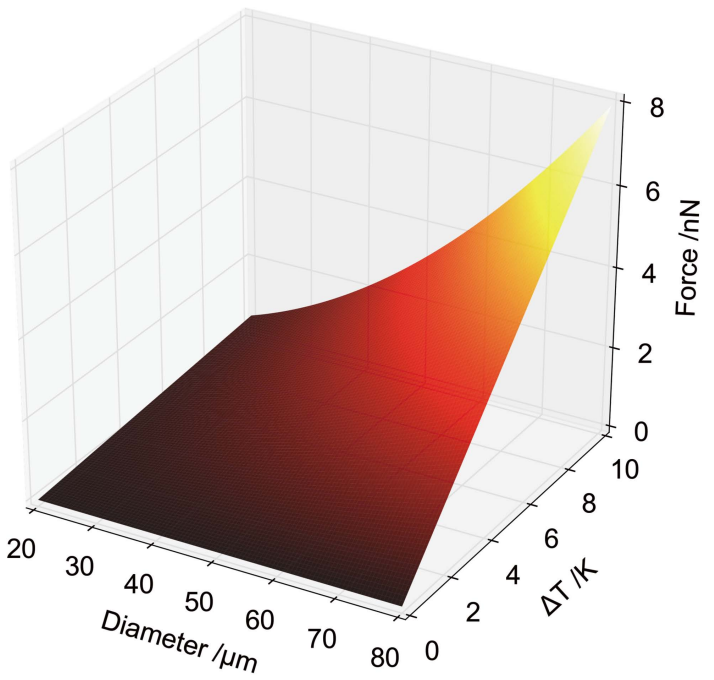

Fig. 9 Driving force map by the photothermal Marangoni effect, derived from Eq. (5). The force of several $\mathrm{nN}$ acts on the droplet under a temperature difference of several K.

Stokes region, where the viscous effect is dominant rather than the inertia. The drag force for the creeping motion of a droplet in fluid, $F_{\mathrm{D}}$, can be presented by the corrected HadamardRybczynski formula, as follows:

$$
F_{\mathrm{D}}=\pi \eta_{\mathrm{c}} d U\left(\frac{2 \eta_{\mathrm{c}}+3 \eta_{\mathrm{d}}}{\eta_{\mathrm{c}}+\eta_{\mathrm{d}}}\right) \kappa,
$$

where $\eta$ is the viscosity of the fluid, and $U$ is the relative velocity of the droplet in a laminar flow of the continuous phase. Subscripts $c$ and $d$ are the continuous and dispersed phase, respectively. $\kappa$ represents the wall correction factor as a function of the blockage ratio, $d / h$ (in an infinite fluid, $\kappa$ is the unity). ${ }^{23}$ We regard $U$ as being the migration velocity in a modified YGB relationship ${ }^{24}$ in which the gravity and the buoyancy are neglected due to the scale effect: ${ }^{25}$

$$
U=\frac{d \lambda_{\mathrm{c}}}{\left(2 \eta_{\mathrm{c}}+3 \eta_{\mathrm{d}}\right)\left(2 \lambda_{\mathrm{c}}+\lambda_{\mathrm{d}}\right)} \cdot \frac{\partial \sigma}{\partial T} \cdot \frac{\partial T}{\partial x},
$$

where $\lambda$ is the thermal conductivity. After $\partial T / \partial x$ is converted to $\Delta T / d$, as described above, the driving force for the droplet by the photothermal Marangoni effect, $F_{\mathrm{M}}$, is consequently described by combined Eqs. (3) and (4) as

$$
F_{\mathrm{M}}=\frac{\pi \eta_{\mathrm{c}} \lambda_{\mathrm{c}}}{\left(\eta_{\mathrm{c}}+\eta_{\mathrm{d}}\right)\left(2 \lambda_{\mathrm{c}}+\lambda_{\mathrm{d}}\right)} \cdot \frac{\partial \sigma}{\partial T} \cdot \Delta T \cdot \kappa d .
$$

By using Eq. (5), we can evaluate the manipulation force for the droplet in the Stokes region. Note that the force is proportional to the temperature gap, $\Delta T$. This tendency agrees with our qualitative prediction (Eq. (2)) in the previous section. The drop size dependence is not simple because the wall correction factor, $\kappa$, is a function of the size. Figure 9 depicts a map of the driving force, $F_{\mathrm{M}}$, as functions of the temperature gap, $\Delta T$, and the droplet size, $d$, under typical experimental ranges in the present study. The properties used in this calculation are shown in Table $1 .^{26-28}$ The magnitude of the force in the present experiment is on the order of $\mathrm{nN}$ under a temperature gap of several $\mathrm{K}$, showing the same order in the previous evaluation. ${ }^{13,15}$ 
Table 1 Properties in the $F_{\mathrm{M}}$ calculation

\begin{tabular}{ccccc}
\hline \multicolumn{2}{c}{ Continuous phase } & & \multicolumn{2}{c}{ Dispersed phase } \\
\cline { 1 - 2 } $\begin{array}{c}\text { Thermal conductivity } \\
\lambda_{\mathrm{d}} / \mathrm{W} \mathrm{m}^{-1} \mathrm{~K}^{-1}\end{array}$ & $\begin{array}{c}\text { Viscosity } \\
\eta_{\mathrm{d}} / \mathrm{mPa} \cdot \mathrm{s}\end{array}$ & & $\begin{array}{c}\text { Thermal conductivity } \\
\lambda_{\mathrm{d}} / \mathrm{W} \mathrm{m}^{-1} \mathrm{~K}^{-1}\end{array}$ & $\begin{array}{c}\text { Viscosity } \\
\eta_{\mathrm{d}} / \mathrm{mPa} \cdot \mathrm{s}\end{array}$ \\
\hline $0.600^{26}$ & $0.894^{26}$ & & $0.199^{27}$ & $29.7^{28}$ \\
\hline
\end{tabular}

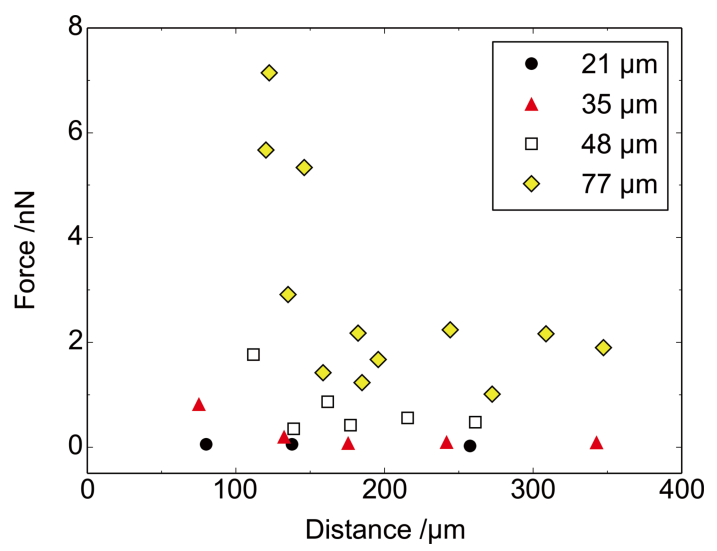

Fig. 10 Relation between the distance from the heating center and the driving force. A shorter distance and a larger size droplet indicate a stronger manipulation force.

This force can be enhanced by adding the larger temperature difference. Figure 10 shows a relation between the distance of the gravity center of the droplet from the heating center and the exerted force based on our model. The experimental data is the same as in Fig. 7. It is obvious that the shorter is the distance and the larger is the droplet size, the larger is the force. The maximum force in this situation is $7.2 \mathrm{nN}$ for the largest droplet of $77 \mu \mathrm{m}$ in diameter at a large temperature difference of $8.6 \mathrm{~K}$. The scattering of the calculated force would be from the error in the temperature measurement. From this result, it can be stated that the model is reasonable to understand the behaviors of controlled droplets. In comparison with other droplet manipulation method i.e., optical tweezers, ${ }^{29}$ the photothermal Marangoni technique in the present study can provide a force more than 1000-times stronger than that of optical tweezers. Although this model can be valid in steady movement because both the Hadamard-Rybczynski and YGB relationships assume no transient behavior, it is valid under low Peclet number condition. Therefore, this model of the manipulation by the photothermal Marangoni convection helps us to estimate the trajectory and limitation of trapping in versatile microfluidic devices with an arbitrary temperature distribution.

\section{Conclusions}

In this paper, our optical approach of picoliter droplet handling controlling local interfacial tension by heating the continuous phase is presented. The theoretical model of the manipulation force for the photothermally controlled droplet can be used to design and optimize the performance of the optical control of the droplet in a microfluidic system. Since our method requires special fabrication or no moving parts in the microfluidic system, flexible and selective droplet-based analysis would be offered.

\section{Acknowledgements}

The authors thank Specified Research Grant in Tokyo University of Science. A part of microfabrication was performed in Center for Nano Lithography \& Analysis, The University of Tokyo, supported by the Ministry of Education, Culture, Sports, Science and Technology (MEXT), Japan. A part of this research was financially supported by a Grant-in-Aid for Young Scientists (A) No. 25709013.

\section{References}

1. J. A. Schwartz, J. V. Vykoukal, and R. C. Gascoyne, Lab Chip, 2004, 4, 11.

2. S.-Y. Teh, R. Lin, L.-H. Hung, and A. P. Lee, Lab Chip, 2008, 8, 198.

3. S. L. Anna, N. Bontoux, and H. A. Stone, Appl. Phys. Lett., 2003, $82,364$.

4. P. Garstecki, M. J. Fuerstman, H. A. Stone, and G. M. Whitesides, Lab Chip, 2006, 6, 437.

5. M. R. Bringer, C. J. Gerdts, H. Song, J. D. Tice, and R. F. Ismagilov, Philos. Trans. R. Soc., A, 2004, 362, 1087.

6. H. N. Joensson and S. H. Andersson, Angew. Chem., Int. Ed., 2012, 51, 12176.

7. D.-K. Kang, M. M. Ali, K. Zhang, E. J. Pone, and W. Zhao, TrAC, Trends Anal. Chem., 2014, 58, 145.

8. D. Chugh, and K. V. I. S. Kaler, Microfluid. Nanofluid., 2010, 8, 445 .

9. D. Lombardi and P. S. Dittrich, Anal. Bioanal. Chem., 2011, 399, 347.

10. R. M. Lorenz, J. S. Edgar, G. D. M. Jeffries, Y. Zhao, D. McGloin, and D. T. Chiu, Anal. Chem., 2007, 79, 224.

11. R. B. Bird, W. E. Stewart, and E. N. Lightfoot, "Transport Phenomena", 2006, John Wiley \& Sons, New York.

12. C. N. Baroud, M. R. de Saint Vincent, and J. P. Delvill, Lab Chip, 2007, 7, 1029.

13. E. Verneuil, M. L. Cordero, F. Gallaire, and C. N. Baround, Langumuir, 2009, 25, 5127.

14. M. L. Cordero, D. R. Burnham, C. N. Baroud, and D. McGloin, Appl. Phys. Lett., 2008, 93, 034107.

15. C. N. Baroud, J. P. Delville, and F. Gallaire, Phys. Rev. E, 2007, 75, 046302.

16. D. Ross, M. Gaitan, and L. E. Locascio, Anal. Chem., 2001, 73, 4117.

17. M. Motosuke, D. Akutsu, and S. Honami, J. Mech. Sci. Technol., 2009, 23, 1813.

18. M. Muto and M. Motosuke, in Proceedings of the 4th Micro and Nano Flows Conference, 2014, London, UK.

19. B. Song and J. Springer, J. Colloid Interface Sci., 1996, 184, 64 .

20. D. C. Duffy, J. C. McDonald, O. J. A. Schueller, and G. M. Whitesides, Anal. Chem., 1998, 70, 4974.

21. J. Lee, W. Shimoda, and T. Tanaka, Meas. Sci. Technol., 2005, 16, 438.

22. R. Savino, A. Cecere, and R. Di Paola, Int. J. Heat Fluid Flow, 2009, 30, 380.

23. J. Chen, Z. Dagan, and C. Maldarelli, J. Fluid Mech., 1991, 233, 405.

24. N. O. Young, J. S. Goldstein, and M. J. Block, J. Fluid Mech., 1959, 6, 350. 
25. P. Tabeling, "Introduction to Microfluidics", 2006, Oxford University Press, UK.

26. Japan Society of Thermophysical Properties (ed.), "Thermophysical Properties Handbook", 2008, Yokendo, Japan.
27. C. L. Yaws, "Handbook of Thermal Conductivity", 1995, Vol. 3, Gulf Professional Publishing, Houston.

28. J. Rabelo, E. Batista, F vio W. Cavaleri, and A. J. A. Meirelles, J. Am. Oil Chem. Soc., 2000, 77, 1255

29. S. C. Kuo and M. P. Sheetz, Science, 1993, 260, 232. 